\title{
A Young Man with Anemia and Recurrent Tachyarrhythmic Episodes
}

\author{
Filippo Patrucco ${ }^{a}$ Maddalena Sarcoli ${ }^{a}$ Renzo Boldorini ${ }^{b}$ Piero Emilio Balbo ${ }^{a}$

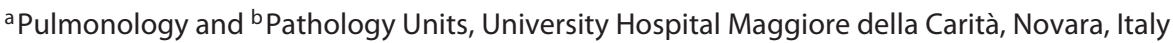

\section{Case Report}

A 22-year-old Caucasian, male, nonsmoking, overweight welfare worker (body mass index 28.7) with personal anamnesis of recurrent hematochezia episodes interpreted as plausible chronic inflammatory bowel disease was admitted to the Emergency Department complaining of general asthenia without any other symptoms. During hospitalization, his tympanic temperature was $36.4^{\circ} \mathrm{C}$, blood pressure was $130 / 70 \mathrm{~mm} \mathrm{Hg}$ and $\mathrm{SpO}_{2}$ $97 \%$ in room air. Physical examination revealed that oral mucosa, ocular conjunctivas and nail beds were pale. Thoracoabdominal objective examination presented regular results. No other abnormalities were reported. Laboratory analysis showed: white blood cells of $6.5 \times 10^{3} / \mu \mathrm{l}$, hemoglobin $(\mathrm{Hb}) 4.9 \mathrm{~g} / \mathrm{dl}$, mean corpuscular volume 60.6 $\mathrm{fl}$, mean corpuscular $\mathrm{Hb}(\mathrm{MCH}) 16.8 \mathrm{pg}, \mathrm{MCH}$ concentration $2.7 \mathrm{~g} / \mathrm{dl}$ and platelets of $351 \times 10^{3} / \mu \mathrm{l}$. The patient was transferred to the Internal Medicine Ward in order to allow an in-depth evaluation. The following laboratory parameters were assessed during hospitalization: serum iron $21 \mu \mathrm{g} / \mathrm{dl}$ (normal values 37-181 $\mu \mathrm{g} / \mathrm{dl}$ ), LDH $377 \mathrm{U} / \mathrm{l}$, normal values for vitamin $\mathrm{D}$ and folic acid, transferrin and ferritin, electrolytes, erythrocyte sedimentation rate
$28 \mathrm{~mm} / \mathrm{h}$, normal total bilirubin, serum creatinine 0.65 $\mathrm{mg} / \mathrm{dl}$, total protein $7.1 \mathrm{~g} / \mathrm{dl}$, normal $\operatorname{IgA}, \operatorname{IgM}$ and $\operatorname{IgG}$ concentrations and serum albumin of $3.4 \mathrm{~g} / \mathrm{dl}$. Anti-gliadin antibody tests were negative, but the IgA endomysium assay and anti-tissue transglutaminase antibody tests were not performed. Urinalysis was negative. A chest Xray showed neither pleural-parenchymal alterations nor cardiomegaly. An electrocardiogram revealed sinus tachycardia. Search for fecal occult blood (in 3 stool samples) was negative. Abdominal echography revealed no alterations or free abdominal effusion. Colonoscopy was unremarkable. In esophagogastroduodenoscopy, there were no stomach lesions but duodenal bleeding due to hemorrhagic telangiectasia; duodenal biopsy evidenced a nonspecific chronic duodenitis pattern without any other structural alterations. ${ }^{99 \mathrm{~m}} \mathrm{Tc}$-labeled red blood cell scintigraphy did not show any pathological accumulation suggestive of intestinal hemorrhage. The patient was given intravenous iron and received 4 units of red blood cell concentrates. At discharge, he had a stable $\mathrm{Hb}$ value of 8.4 $\mathrm{g} / \mathrm{dl}$.

During the following years, the patient was in good clinical condition and only suffered from exercise-induced dyspnea [MMRCDS (Modified Medical Research

\section{KARGER}

E-Mail karger@karger.com

www.karger.com/res
(C) 2013 S. Karger AG, Basel

0025-7931/13/0862-0149\$38.00/0
Piero Emilio Balbo

Pulmonology Unit, University Hospital Maggiore della Carità

Corso Mazzini No. 18

IT-28100 Novara (Italy)

E-Mail piero.balbo@libero.it 


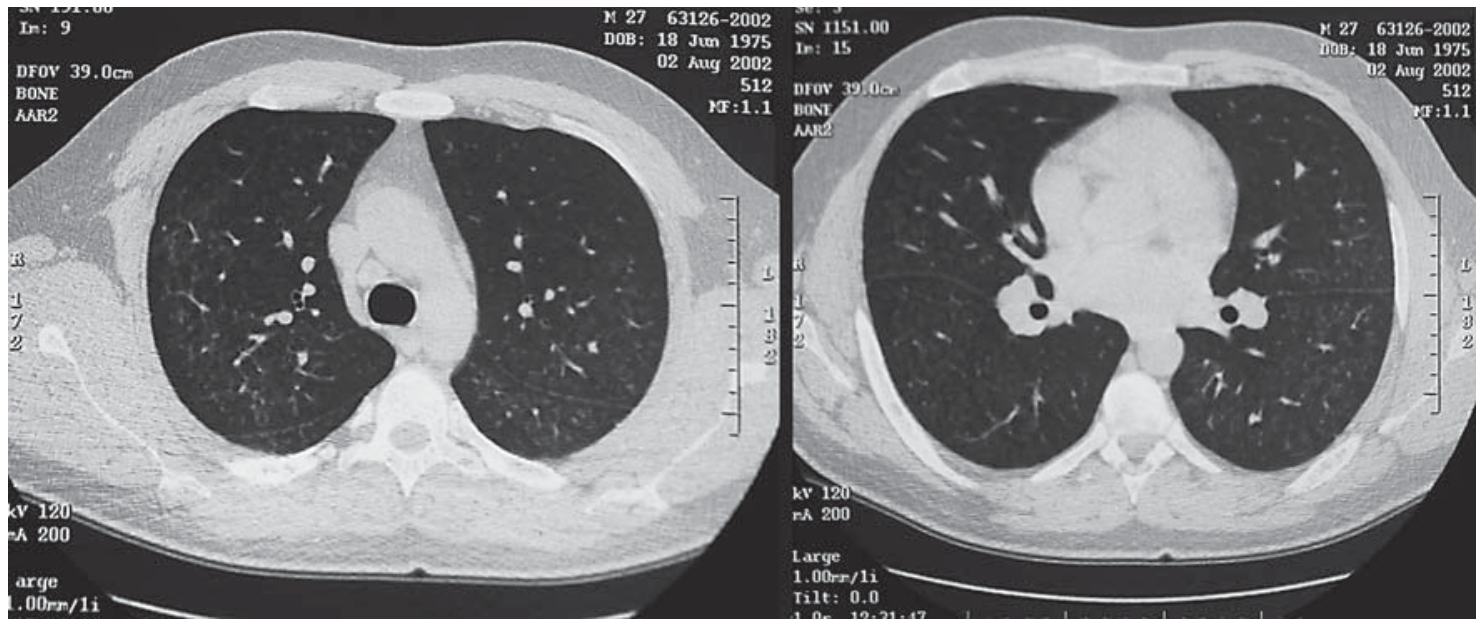

Fig. 1. CT images of the chest. Parenchymal window setting shows a reticular and nodular bilateral pattern without any other alterations.
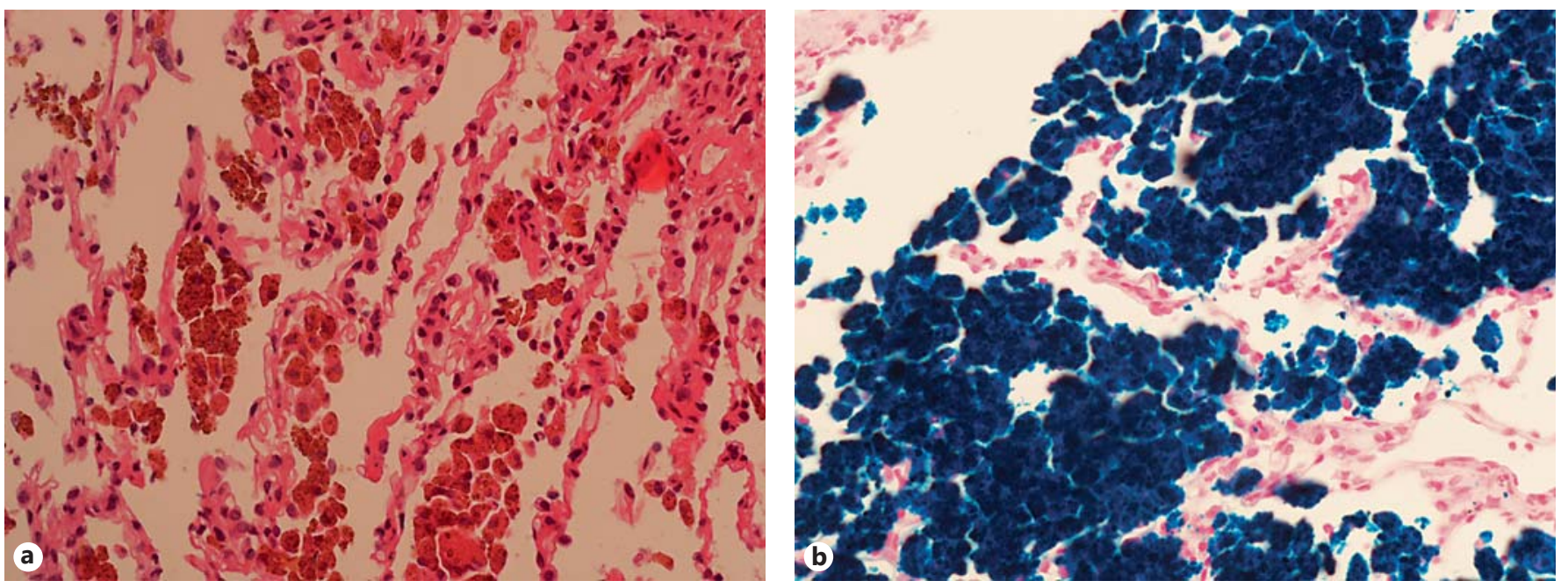

Fig. 2. Histologic section of lung biopsy showing massive accumulation of strongly pigmented macrophages without evidence of capillaritis $(\mathbf{a} \mathrm{H} \& \mathrm{E}$, original magnification $\times 40)$. Pearls staining confirming iron depositions (b pearls, original magnification $\times 20$ ).

Council Dyspnea Scale) 1]. At the age of 27 years, he was admitted to the Pulmonology Department after repeated hemoptysis. Computed tomography revealed a reticular, nodular bilateral pattern (fig. 1). Bronchoalveolar lavage and transbronchial lung biopsy presented cyto- and histopathological alterations compatible with idiopathic pulmonary hemosiderosis (IPH; fig. 2). Screening for autoimmune disorders and serological tests for IgA antitissue transglutaminase and endomysial antibodies were negative. Prednisone-based treatment was started ( $1 \mathrm{mg} /$ $\mathrm{kg} /$ day), which was tapered off to a maintenance dose of $5 \mathrm{mg} /$ day despite the persistence of mild dyspnea (MMRCDS 1).

Two years later, at the age of 29 , he was admitted to hospital after an atrial fibrillation episode, which deteriorated into ventricular, well-tolerated tachycardia; intracardiac electrophysiological study revealed an easy inducibility for atrial fibrillation and ventricular tachycar- 
dia (200 b.p.m.), left bundle branch block and sinus node conduction dysfunction. Subsequent myocardial scintigraphy showed reduced uptake without signs indicative of coronary pathology. Cardiac magnetic resonance imaging revealed some characteristics suggesting multiple myocarditis foci. At discharge, his treatment included amiodarone and a $\beta$-blocker.

One year later, he was admitted once again after an atrial fibrillation and ventricular tachycardia, which resolved spontaneously; an electrocardiogram confirmed atrial fibrillation associated with a left bundle branch block. Echocardiography revealed mild left ventricular dilatation and mild mitral regurgitation (ejection fraction: 57\%). At discharge, he was advised to continue the previously prescribed pharmacologic treatment.

One year after his last hospital stay, he was admitted to the Emergency Department complaining of worsening dyspnea (MMRCDS 4) and hemoptysis. On physical examination, he was tachycardiac (115 bpm.), tachypneic (respiratory rate 26 breaths/min) and pale but without other abnormalities except for the presence of mild leg swelling and abdominal distension (diarrhea and flatu- lence were absent). Laboratory analysis revealed anemia $(\mathrm{Hb} 10.1 \mathrm{~g} / \mathrm{dl}$, mean corpuscular volume $65.9 \mathrm{fl}, \mathrm{MCH}$ $19.6 \mathrm{pg}, \mathrm{MCH}$ concentration $29.7 \mathrm{~g} / \mathrm{dl}$ ) but no other alterations. Electrocardiographic examination showed sinus tachycardia with isolated supraventricular ectopic beats. A chest X-ray disclosed a reticular interstitial pattern and hilar congestion. The patient was admitted to the Pulmonology Department; laboratory analysis confirmed anemia (serum iron $<10 \mu \mathrm{g} / \mathrm{dl}$, transferrin $244 \mathrm{mg} / \mathrm{dl}$, ferritin $61.5 \mathrm{ng} / \mathrm{dl}$, reticulocytes $23.5 \times 10^{3} / \mu \mathrm{l}$ ); HIV, HBV and HCV were negative, and, for the first time, we noted a reduction in the total protein concentration $(5.7 \mathrm{mg} / \mathrm{dl})$.

Fibrobronchoscopy and bronchoalveolar lavage showed hemorrhagic alveolitis. Treatment with prednisone $(1 \mathrm{mg} / \mathrm{kg} /$ day $)$ and azathioprine $(2 \mathrm{mg} / \mathrm{kg} /$ day $)$ was started. Echocardiography revealed dilatative myocardiopathy with $24 \%$ ejection fraction. In respiratory function tests, forced vital capacity (FVC) was $69.8 \%$, forced expiratory volume in $1 \mathrm{~s}\left(\mathrm{FEV}_{1}\right) 66.7 \%, \mathrm{FEV}_{1} / \mathrm{FVC} 0.95$ and single-breath diffusion lung capacity for CO $57.7 \%$ of predicted.

What is your diagnosis? 


\section{Diagnosis: IPH Associated with Dilated Cardiomyopathy and Celiac Disease}

The patient was further investigated. Particular attention was paid to the reevaluation of his autoimmunity profile; anti-gliadin, anti-endomysial and anti-transglutaminase $(>128 \mathrm{AU} / \mathrm{ml})$ antibody tests were positive. Following esophagogastroduodenoscopy and duodenal mucous membrane biopsy, celiac disease (CD) was diagnosed. He was immediately put on a gluten-free diet (GFD) and, during the following 3 months, he was gradually tapered off immunosuppressive treatment and thus his clinical status improved. Two years after GFD initiation, magnetic resonance imaging evidenced substantial stability of hemosiderin deposits, and ejection fraction was markedly improved on echocardiography (51\%). Pulmonary function tests were also better: $\mathrm{FEV}_{1} 79.9 \%$, FVC $80.1 \%, \mathrm{FEV}_{1} / \mathrm{FVC} 0.99$ and single-breath diffusion lung capacity for CO $76.9 \%$ of predicted. After another 2 years, a further improvement in ejection fraction (57\%) was recorded, a condition which allowed anti-arrhythmic drugs to be discontinued. At present, the patient's $\mathrm{Hb}$ is $15.9 \mathrm{~g} / \mathrm{dl}, \mathrm{FEV}_{1} 112 \%$, FVC 110\%, single-breath diffusion lung capacity for CO $96.7 \%$ of predicted and ejection fraction $60 \%$ (table 1 ).

IPH is a rare disorder of unknown etiopathogenesis; it is characterized by recurrent episodes of a triad of symptoms comprising diffuse alveolar hemorrhage, hemoptysis and iron deficiency anemia. Symptoms usually appear during the 1st decade, and less commonly during the 3rd or 4th [1]. Diagnosis of IPH is based on bronchoalveolar lavage and lung biopsies indicative of hemosiderin-laden macrophages in alveolar fluid and alveoli, respectively, without histological or immunohistochemical evidence of capillaritis. Other potential causes of alveolar hemorrhage have to be excluded first before further investigations, ranging from serological to microbiological and radiological tests, are performed $[2,3]$. In the literature, average survival is highly variable, but in children and adolescents the disease tends to take a rapid and progressive course. In adults, progression of disease is typically characterized by less pronounced symptoms and a more favorable prognosis [4].

The association between IPH and CD has been reported in both children and adults $[4,5]$; the pathogenic link between these two diseases is unclear despite the suspicion of immunological correlations. Currently, there are three pathogenetic hypotheses that may explain this correlation: the presence and accumulation of immune complexes in the basement membrane of alveolar capillaries originating from food allergens, cross-reactivity between anti-reticulin antibody and the alveolar basement membrane or a viral cause involving adenovirus 12 and its association with CD [6].

The regression of pulmonary symptoms is related to the introduction of GFD $[4,5]$, even though some observations suggest a disguising action of immunosuppressive drugs employed to treat IPH [7]. Khemiri et al. [7] confirmed the beneficial effect of GFD in a case of relapse of pulmonary symptoms after the reintroduction of gluten in the diet. Pacheco et al. [8] reported long-term remission of pulmonary symptoms with GFD. Our case report confirms that the exclusion of gluten from the patient's diet enabled to progressively decrease immunosuppressive drug treatment, and thus his hematologic, cardiologic and respiratory parameters improved.

In the literature, data on the timing of immunosuppressive treatment cessation after GFD initiation are lacking; it is advisable to wean patients off immunosuppressive drugs after pulmonary symptoms do not present for a predetermined time period. Subsequent follow-up is suggested in order to guarantee the reintroduction of the immunosuppressive treatment in case symptoms relapse [9].

An association between dilated cardiomyopathy and CD has been stated in the literature [10]; a recent study reported increased prevalence of CD (5.8\%) in patients with dilated cardiomyopathy [11]. Malabsorptive, immunological and infectious causes were proposed to explain the correlation between both diseases [12-14]. It has been demonstrated that the introduction of GFD may improve ejection fraction; some patients benefit exclusively from a diet therapy associated with the discontinuation of the immunosuppressive treatment [9]. Our case report confirms such an approach with the recovery of ejection fraction with values $>50 \%$ through a 4 -year term and the complete discontinuation of anti-arrhythmic drugs. We observed that the immunosuppressive treatment was adequate to control pulmonary symptoms despite the persistence of mild dyspnea; complete control of cardiologic symptoms and arrhythmic episodes was only achieved after the start of GFD.

Our case report highlights some noteworthy key elements focusing on these three linked conditions. Firstly, we present a rare case of IPH in a young adult, inasmuch as the majority of IPH cases are encountered in children; in the literature, you can find similar episodes, although they are extremely rare. 
Table 1. Patient history

\begin{tabular}{lllll}
\hline $\begin{array}{l}\text { Patient age } \\
\text { years }\end{array}$ & \multicolumn{2}{l}{ Main clinical events } & & \multirow{2}{*}{ Therapy } \\
\cline { 2 - 4 } & constitutional & respiratory & cardiac & \\
\hline 22 & $\begin{array}{l}\text { anemia }(\mathrm{Hb} 4.9 \mathrm{~g} / \mathrm{dl}), \\
\text { asthenia }\end{array}$ & none & none & blood transfusions, intravenous iron \\
\hline 27 & anemia $(\mathrm{Hb} 9.4 \mathrm{~g} / \mathrm{dl})$ & dyspnea, hemoptysis & none & prednisone \\
\hline 30 & anemia $(\mathrm{Hb} 11.9 \mathrm{~g} / \mathrm{dl})$ & dyspnea & arrhythmia & prednisone, amiodarone, $\beta$-blockers \\
\hline 31 & anemia $(\mathrm{Hb} 10.8 \mathrm{~g} / \mathrm{dl})$ & dyspnea & arrhythmia & prednisone, amiodarone, $\beta$-blockers \\
\hline 33 & $\begin{array}{l}\text { anemia }(\mathrm{Hb} 10.1 \mathrm{~g} / \mathrm{dl}), \\
\text { hypoproteinemia }\end{array}$ & dyspnea, hemoptysis & none & prednisone, azathioprine, amiodarone, \\
\hline
\end{tabular}

An unusual point of interest is that our patient was investigated with esophagogastroduodenoscopy and for the presence of specific antibodies for $\mathrm{CD}$ which resulted negative; he developed CD in a second step when IPH was already diagnosed and treated. In the literature, this sequence is quite sporadic, because we usually observe the presence of CD before or concomitant with the development of IPH.

Another significant issue is related to the fact that the literature is used to describe cases of CD associated with dilated cardiomyopathy, whereas our case report cannot prove that the cardiac involvement is linked either to CD or to hemochromatosis; we can only hypothesize that these three pathologies are correlated, and that an immunologic mechanism is probably responsible at least in part for the pathological multiorgan involvement. The hypothesis that IPH, CD and cardiomyopathy are linked is suggested by the improvement in respiratory, cardiologic and laboratory function after the introduction of GFD; most likely the main role is played by $\mathrm{CD}$, since the sole introduction of GFD was sufficient to taper off cardiologic and immunosuppressive treatment. This hypothesis may be confirmed by the long follow-up period without any other symptomatic episode.
We are still dubious about the real cause of the first anemia episode as the data in our files cannot completely explain the original signs. We can argue in favor of a wrong interpretation of the histological specimens (unavailable for reexamination) or a false-negative anti-gliadin antibody assay. Alternatively, other potential causes of sideropenic anemia should be borne in mind.

In conclusion, cases of IPH and CD have already been reported in the literature and the absence of symptoms after the introduction of GFD recorded in our patient confirms the previously published data. Long-term survival, cardiac involvement and our patient's mature age make this rare case an interesting issue to study the correlation between such pathologies.

\section{Key Words}

Anemia - Celiac disease - Dilated cardiomyopathy · Idiopathic pulmonary hemosiderosis

\section{References}

Wright PH, Menzies IS, Punder RE, Keeling PW: Adult idiopathic pulmonary hemosiderosis and celiac disease. QJ Med 1981;197: 95-102.

-2 Milman N, Møller Pedersen F: Idiopathic pulmonary hemosiderosis. Epidemiology, pathogenic aspects and diagnosis. Respir Med 1998; 92:902-907.
Picard C, Cadranel J, Porcher Prigent H, et al: Alveolar haemorrhage in the immunocompetent host: a scale for early diagnosis of an immune cause. Respiration 2010;80:313-320.

4 Malhotra P, Aggarwal R, Aggarwal AN, Jindal SK, Awasthi A, Radotra BD: Coeliac disease as a cause of unusually severe anaemia in a young man with idiopathic pulmonary haemosiderosis. Respir Med 2005;99:451-453. 
5 Reading R, Watson JG, Platt JW, Bird AG: Pulmonary haemosiderosis and gluten. Arch Dis Child 1987;62:513-515.

6 Perelman S, Dupuy C, Bourrillon A: The association of pulmonary hemosiderosis and celiac disease. Apropos of a new case in a child (in French). Ann Pediatr (Paris) 1992;39:185188.

7 Khemiri M, Ouederni M, Khaldi F, Barsaoui S: Screening for celiac disease in idiopathic pulmonary hemosiderosis. Gastroenterol Clin Biol 2008;32:745-748.
8 Pacheco A, Casanova C, Fogue L, Sueiro A: Long-term clinical follow-up of adult idiopathic pulmonary hemosiderosis and celiac disease. Chest 1991;99:1525-1526.

-9 Sethi GR, Singhal KK, Puri AS, Mantan M: Benefit of gluten-free diet in idiopathic pulmonary hemosiderosis in association with celiac disease. Pediatr Pulmonol 2010;46:302305.

10 Goel NK, McBane RD, Kamath PS: Cardiomyopathy associated with celiac disease. Mayo Clin Proc 2005;80:674-676.

11 Curione M, Barbato M, De Biase L, Viola F, Lo Russo L, Cardi E: Prevalence of coeliac disease in idiopathic dilated cardiomyopathy. Lancet 1999;354:222-223.
2 Chimenti C, Pieroni M, Maseri A, Frustaci A: Dilated cardiomyopathy and celiac disease. Ital Heart J 2002;3:385

13 van Elburg RM, Uil JJ, Mulder CJ, Heymans HS: Intestinal permeability in patients with coeliac disease and relatives of patients with coeliac disease. Gut 1993;34:354-357.

14 DeMeo MT, Mutlu EA, Keshavarzian A, Tobin MC: Intestinal permeation and gastrointestinal disease. J Clin Gastroenterol 2002;34: 385-396. 